\title{
Aptamer-Mediated Cancer Gene Therapy
}

Dongxi Xiang $^{1^{*}}$, Sarah Shigdar ${ }^{1 *}$, Greg Qiao ${ }^{2}$, Shu-Feng Zhou ${ }^{3}$, Yong ${ }^{4}{ }^{4}$, Ming Q Wei ${ }^{5}$, Liang Qiao ${ }^{6}$, Hadi Al.Shamaileh ${ }^{1}$, Yimin $\mathrm{Zhu}^{7}$, Conglong Zheng ${ }^{8}$, Chunwen $\mathrm{Pu}^{9 * *}$ and Wei

\section{$\operatorname{Duan}^{1 * *}$}

${ }^{1}$ School of Medicine, Deakin University, Pigdons Road, Waurn Ponds, Victoria, 3217, Australia.

${ }^{2}$ Department of Chemical and Biomolecular Engineering, Melbourne School of Engineering

The University of Melbourne, Parkville, Victoria 3010, Australia

${ }^{3}$ Department of Pharmaceutical Sciences, College of Pharmacy, University of South Florida, Tampa, FL 33612, USA.

${ }^{4}$ Cancer Care Centre, St George Hospital, Kogarah, NSW2217, and St George and Sutherland Clinical School, University of New South Wales (UNSW), Kensington, NSW2052, Australia

${ }^{5}$ Division of Molecular and Gene Therapies, Griffith Health Institute and School of Medical Science, Griffith University, Gold Coast, QLD 4222, Australia

${ }^{6}$ Storr Liver Unit, at the Westmead Millennium Institute, the University of Sydney at the Westmead Hospital, Westmead NSW, 2145, Australia

${ }^{7}$ Suzhou Key Laboratory of Nanobiomedicine, Division of Nanobiomedicine, Suzhou Institute of Nano-Tech and Nano-Bionics, Chinese Academy of Sciences, Suzhou, Jiangsu, China, 215123

${ }^{8}$ Department of Biology, Medical College, Dalian University, Liaoning, People's Republic of China

${ }^{9}$ The Affiliated Zhongshan Hospital of Dalian University, 6 Jiefang Road, Dalian, Liaoning, The People's Republic of China, 116001. 
* These authors contributed equally.

** Corresponding authors: E-mail: dahaixiaowen@163.com (C. Pu); or wduan@deakin.edu.au (W. Duan). 


\begin{abstract}
Cancer as a genetic disorder is one of the leading causes of death worldwide. Conventional anticancer options such as chemo- and/or radio-therapy have their own drawbacks and could not provide a cure in most cases at present. More effective therapeutic strategies with less side effects are urgently needed. Aptamers, also known as chemical antibodies, are single strand DNA or RNA molecules that can bind to their target molecules with high affinity and specificity. Such site-specific binding ability of aptamers facilitates the delivery and interaction of exogenous nucleic acids with diseased genes. Thus, aptamer-guided gene therapy has emerged as a promising anticancer strategy in addition to the classic treatment regimen. Aptamers can directly deliver anti-cancer nucleic acids, e.g. small interfering RNA, micro RNA, anti-microRNA and small hairpin RNA, to cancer cells or function as a targeting ligand to guide nanoparticles containing therapeutic nucleic acids. This review focuses on recent progress in aptamer-mediated gene therapy for the treatment of hepatocellular carcinoma and other types of cancers, shedding light on the potential of this novel approach of targeted cancer gene therapy.
\end{abstract}




\section{INTRODUCTION}

Cancer is a multifaceted disease characterized by inter- and intra-patient genomic and cellular heterogeneity, arising from the long-term accumulation of genetic mutation and/or epigenetic alterations [1]. Cancer is a leading cause of death worldwide, with 1,665,540 new cases and 585,720 deaths predicted in the United States of America alone in 2014 [2]. Thus, the exploration of alternative and more efficacious approaches for eradicating cancer is urgently needed. Cancer gene therapy emerges to be a promising strategy to combat cancer.

Gene therapy aims to introduce therapeutic genetic materials (oligonucleotides, DNAs or RNAs) into the diseased cells/tissues or an organ to correct genetic errors [3, 4]. Gene therapy for cancer has been the most attractive area in this research field with two-thirds of gene therapies trials focusing on cancer therapy [5-7]. Molecularly targeted therapies entail inhibiting oncogenes, restoring the function of tumour suppressor genes or the introduction of suicide genes/prodrug systems [7]. The success of cancer gene therapy largely depends on the selective delivery of sufficient therapeutic dose of nucleic acids into target tumour cells. To achieve this aim, a great number of sophisticated tools have been engineered, which can be divided into two major classes - viral vectors and non-viral vectors (i.e. liposomes, oligonucleotides, DNA or RNA multiplexes) [8, 9]. However, these vectors still face several challenges such as immune response, insertional mutagenesis and lack of specificity of transgene delivery [7].

Aptamers, also known as, chemical antibodies are single-stranded oligonucleotides and bind to their targets through shape complementarity with high affinity and specificity in a wide array of clinical applications [10-12]. Being generated by chemical synthesis, aptamers can be easily modified for desired applications, e.g. attaching to therapeutic drugs, nanoparticles and imaging agents without loss-of-function [13, 14]. In addition, upon binding to their cell surface ligands, aptamers together with the conjugated nucleic acids carrier can rapidly internalise into cells via receptor-mediated endocytosis. After internalisation, aptamers can direct exogenous therapeutic genes to the cytoplasm or nuclei to enhance the efficacy of cancer gene targeting and/or genetic modification [15, 16]. Thus, these attributes make aptamers promising choices for molecular recognition and gene delivery in targeted cancer therapy. The current review will update recent progress of aptamer-guided gene targeting in cancer therapy, with hepatocellular carcinoma (HCC) as a prime example to illustrate the principles and capabilities that can be applied to other types of cancer. 


\section{AN OVERVIEW on the TARGETED CANCER GENE THERAPY}

Gene therapy is a therapeutic modality in which nucleic acids are used as therapeutic agents and selectively delivered into patient's cells with minimal toxicity $[4,17]$. The functionalised therapeutic genes aim to correct genetic errors by inhibiting defective gene expression or repairing the mutated gene. Among genetic diseases, cancer is the most studied case in gene therapy for clinical trials as gene therapy is considered to be a promising and rational approach of combating cancer [7]. Because of the complexity of tumour, several effective gene therapy strategies have been investigated for treatment of malignant tumour. These strategies can be categorised into two approaches: immunologic and molecular avenues [18]. Transfection of immune-stimulant genes such as cytokines (i.e., Interleukin-12) into cancer cells or engineering antigen presenting cells with tumour-associated antigens are commonly adopted approaches in cancer immunologic-based gene therapy [19]. As for molecular approaches in cancer gene therapy, blockage of cellular oncogenes, restoration of tumour suppressor gene and introduction of genes encoding enzymes that can convert non-toxic substances to toxic agent (suicide gene) are three most frequently pursued approaches [20]. Other effective strategies of molecular cancer gene therapy entail the introduction of antiangiogenic genes into tumour vasculature, expression of suicide genes coupled with otherwise non-toxic chemotherapeutic agent and inhibiting multi-drug resistance genes in tumour cells [20]. In 2003, the first commercially produced gene based cancer therapy was manufactured by Shenzhen SiBiono GeneTech. Gendicine ${ }^{\mathrm{TM}}$, a replication-incompetent recombinant human Ad5-p53, was approved by the Chinese State Food and Drug Administration to treat head and neck squamous cell carcinoma [21]. Again in 2006, Shanghai Sunway Biotech, commercially released a conditionally replicative adenovirus therapy, Oncorine ${ }^{\mathrm{TM}}$ [22]. However, it is fair to say that cancer gene therapy has yet to realise its full potential.

Successful cancer gene therapy relies on the efficient delivery of therapeutic genes into tumour cells with high specificity. Non-viral gene delivery has several advantages, including relative ease for large-scale production and reproducibility, with much enhanced safety over viral methods. This is because viral delivery remains a safety concern as a viral administration may enhance the risk of host immune response with subsequent adverse effects occurring $[8,9]$. Although viral vectors are generally more efficient in gene delivery than their non-viral compartments, virus can only carry very limited gene. Furthermore, insertional mutagenesis caused by the viral vector poses an additional risk to the patient [23]. 
Recent technological advances herald new generations of smart non-viral vectors that could yield high gene transfer efficiencies similar to their viral compartments for liver hepatocellular gene therapy [24]. Among those non-viral carriers, aptamer has emerged as one of the promising non-viral gene delivery systems $[25,26]$.

The anatomic characteristics of the liver render it as an ideal therapeutic target for gene therapy. Being the only organ with two circulation systems in the body, the liver is extensively vascularized that hepatocytes and liver parenchyma are readily accessible to macromolecules such as DNA fragments or viral particles [27]. These factors are import for gene therapy applications for liver malignancies. Here we focus on recent advances in aptamer-mediated cancer gene therapy for the treatment of hepatocellular carcinoma (HCC), one of the common and aggressive types of liver cancer [28, 29].

\section{APTAMER-GUIDED CANCER GENE THERAPY}

\section{Aptamer and Advanced Properties}

Aptamers, single-stranded DNA or RNA oligonucleotides, derive their name from the Latin word 'aptus' meaning 'to fit'. They can be selected in vitro by a process known as systematic evolution of ligands by exponential enrichment (SELEX) (Figure 1) [30, 31]. The targets for aptamers include nucleic acids, peptides, proteins, organic and inorganic molecules, or even a whole cell $[10,11]$. Due to its nature of in vitro selection, the SELEX can be used to select/isolate aptamers with extreme high affinity (e.g. a $K_{D}$ of $1 \mathrm{pM}$ ) as well as high specificity. Aptamers fold through intra-molecular interactions to form tertiary conformations that recognize and bind targets via shape complementarity. Once the sequence of an aptamer is confirmed, it can be synthesized with high reproducibility and purity in vitro at a relatively low cost, which is a key advantage for drug development [14]. Antibodies differ from aptamers in that they are produced and purified from animals or cell culture with their binding characteristics being influenced by conditions of each animal and cultured cells. In contrast, aptamers are manufactured by chemical synthesis in vitro without the need of using animals or cells and thus possess much less batch-to-batch variations. Also, being composed of nucleic acids, aptamers are much more stable at room temperature and in nonphysiological conditions. Thus, unlike antibodies, the stability of aptamers allows them to be stored stably across a wide range of storage and temperature conditions without the need of stabilization aids, as aptamers can be easily re-folded to assume their 3-D structure by one cycle of heating and cooling $[32,33]$. Furthermore, aptamers have been shown to have low 
immunogenicity and are non-toxic [14]. Critically important for the application for cancer therapy, aptamers can penetrate into the core of the tumour much more efficiently than antibodies as they are $20-25$-fold smaller than a full sized monoclonal antibody [34, 35]. Nonetheless, there are several limitations of nucleic acid aptamers when they were functioned in vivo through the blood circulation. Being polynucleotides, aptamers are exposed to enzyme degradation by exo- and/or endo-nucleases, resulting in a reduction of in vivo circulatory half-life. This problem can be addressed by incorporating unnatural nucleotide bases (locked and unlocked nucleic acids), introducing backbone or chemical modifications to aptamers, thus reducing the susceptibility to the exposure of endonuclease and exonuclease $[13,36,37]$. Limit residence time in blood is another challenge with in vivo aptamer applications, which is largely because of fast removal of aptamer by renal filtration as the size of most aptamers is smaller than the renal filtration threshold of $40 \mathrm{kDa}$ [37]. Judicial modification of aptamers with polyethylene glycol (PEG) can increase aptamer's blood resident time and thus enhance tumour delivery [38]. Table $\mathbf{1}$ summarizes key features aptamers in comparison with antibodies.

\section{Principle of Aptamer-Guided Gene Therapy}

Unlike antibodies that can be easily denatured or inactivated because of the rising acidity in the vesicles/organelles down the endocytic pathway, aptamers engineered with chemical modifications remain stable and functional after endocytosis [39-44]. In addition to functioning as drug delivery carriers, aptamers have been actively exploited as a gene delivery vehicle for small interfering RNA (siRNA), micro RNA (miRNA) and short hairpin RNA (shRNA) [13, 45-47]. These aptamer-guided nucleic acids can be delivered in vivo via multiple routes such as intratumoural injection, intravenous injection, and percutaneous injection. Once internalized, aptamers interact with the host DNA and/or RNA to alter the gene expression of targeted malignant cells. Aptamer can medicate gene therapy through either the immunologic or the molecular avenues. Intense effort has been devoted to explore aptamers as an effective gene therapy modality for cancer therapeutics [48-50].

Gene targeting is a powerful tool in functional analysis of genes. However, the application of targeted gene modification in gene therapy is hampered by its low frequency, i.e. $1 \times 10^{-5}-10^{-}$

7, in many organisms. Recent work from Ruff and colleagues provided an elegant demonstration for the power of aptamers in repairing a genomic locus [51]. They developed a novel aptamer-based gene targeting approach for gene correction, in which a DNA aptamer linked with an exogenous oligonucleotide of 40-54 bases was delivered to the close vicinity 
of the genomic locus to efficiently repair the target gene. Specifically, the authors generated an aptamer against the homing endonuclease, site-specific meganuclease I-SceI, to deliver the tethered donor DNA for gene correction close to the site of the I-SceI DNA double-strand break. With the I-SceI aptamer, the donor molecule for gene correction is brought to the vicinity of its target site to enhance the specificity of efficacy of genetic modification. Indeed, this aptamer-guided gene targeting approach has enhanced gene targeting up to 16-fold in human cells [51]. Although this was accomplished in vitro using plasmid transfection, this work represents a novel proof-of-principle concept, where aptamers can be used as tools for increasing gene targeting efficiency for oncogenes and modulating overexpressed signalling or regulatory genes in cancer.

\section{Free Aptamer-Mediated Inhibition of Hepatocellular Tumour Metastasis}

The failure of cancer treatment and poor prognosis with $\mathrm{HCC}$ is often due to tumour recurrence and metastasis after initial surgical resection or chemotherapeutic treatment [28, 29]. To date, sorafenib, also known as nexavar, is the only chemotherapy agent approved for the treatment of advanced primary liver cancer [52]. There are also ongoing clinical trials for HCC treatment such as brivinib (an inhibitor of FGFR1 and VEGFR1), doxorubicin, bevacizumab and ABT-869 (a receptor tyrosine kinase inhibitor of VEGF), but most of them lack specificity and have limited efficacy [53]. A DNA methyltransferase inhibitor, SGI-110, used as epigenetic therapy for cancer has entered Phase 2 clinical trial for advanced HCC patients who failed prior treatment with sorafenib [54]. The final completion date for primary outcome measurement of SGI-110 is expected by October 2014 [54]. We are awaiting novel targeted therapy with improved efficacy.

Osteopontin (OPN), a secreted glycophosphoprotein overexpressed in malignant cells, is a potential prognostic marker and a therapeutic target for HCC invasion, progression and metastasis, it is also capable of promoting epithelial-mesenchymal transition (EMT) for HCC migration [55-57]. Studies from independent groups have shown that increased serum/plasma osteopontin level is highly associated with poor clinical prognosis and treatment failure of HCC after treatment with curative intent, suggesting that blockage of OPN function could significantly reduce HCC growth and progression [53, 55, 57-60]. Being an extracellular protein, OPN is one of the ideal therapeutic targets for aptamer-based therapy. Zhao and colleagues [61] engineered polyethylenimine nanoparticles (M-PEI) to carry a short-hairpin RNA (shRNA) for targeting and reducing OPN expression both in vitro and in vivo using a HCC-LM3 xenograft model. The nano-encapsulation of shRNA could 
circumvent the nuclear degradation and sustain the duration of M-PEI-carried shRNA in vivo. The studies confirmed that OPN facilitated tumourigenesis and metastasis by protecting tumour cells from apoptosis. Down-regulation of OPN-induced or/and mitochondriamediated HCC apoptosis coupled with the improved sensitivity of shRNA-transfected HCC cells to chemotherapeutic drugs resulted in the inhibition of HCC cell growth, adhesion and invasion in vitro, as well as suppression of tumourigenicity and lung metastasis in vivo. In addition, Talbot's group utilised a 2'-O-methoxy (2'-OMe) modified RNA OPN aptamer of 40 bases (OPN-R3) with a $K_{\mathrm{d}}$ of $57.2 \mathrm{nM}$ for the blockade of extracellular OPN in HCC to evaluate the antitumour properties [53,62]. Analysis of anti-tumour function both in in vitro and ex vivo settings revealed a significant reduction of EMT by this aptamer [53]. Moreover, following the establishment of an in vivo xenotransplant model of Sk-Hep-1 HCC, the OPN aptamer or control aptamer $(0.5 \mathrm{mg} / \mathrm{kg})$ were administrated i.v. every 2 days from week 3 to week 8 . In the course of 8 weeks, OPN aptamer inhibited tumour growth by more than 10fold compared with that of untreated or control-aptamer treated mice. If the efficacy of OPN aptamers can be confirmed in patient-derived xenografts to demonstrate their ability to significantly decrease EMT marker expression and inhibit the HCC growth, aptamer-guided inhibition of OPN will be a promising therapeutic option for HCC.

Carcinoembryonic antigen (CEA) is overexpressed in the majority of carcinomas including colon, lung, mucinous ovarian and endometrial carcinomas [63]. Being an important tumour marker for colorectal cancer, CEA overexpression is known to promote hepatic metastasis of colon cancer cells [64]. It has been revealed that five amino acids (PELPK) in the hinge region is required for binding to the CEA receptor, heterogeneous nuclear ribonucleoprotein M4, on Kupffer cells and is thus associated with the initiation process of hepatic metastasis [65]. In order to inhibit hepatic metastasis of colon cancer cells, Lee and co-workers have generated an RNA aptamer with 49 nucleotides in length, YJ-1, that specifically binds to the PELPK motif in CEA [41]. Upon binding specifically to CEA-positive cells, the CEA RNA aptamers inhibited interactions between CEA and heterogeneous nuclear ribonucleoprotein M4, homotypic aggregation, migration, as well as invasion by CEA-positive cancer cells, but did not affect adhesion of endothelial cells. In addition, the CEA aptamer induced colon cancer cell anoikis by interrupting the interaction between death receptor 5 and CEA. In a LS174T xenograft colon cancer model, two million of CEA-positive LS174T cells were preincubated with CEA aptamers for $5 \mathrm{~min}$ before being splenetically injected. Amazingly, when the animals were sacrificed on the $35^{\text {th }}$ day after injection, there was a $91 \%$ reduction in total tumour volume of hepatic metastasis in the CEA aptamer-treated group compared with 
the control. Thus, the CEA aptamer is a promising lead for future development of an agent capable of inhibiting migration and invasion by colonrectal cancer cells, and preventing hepatic metastasis.

\section{Aptamer-siRNA Chimera for Cancer Gene Targeted Therapy}

Small interfering RNA (siRNA) can be incorporated into the RNA-induced silencing complex (RISC), resulting in the degradation of target mRNA, based on the pathway known as RNA interference (RNAi) [66]. Despite the potential of specifically inhibiting gene expression of unlimited targets with high efficiency, the inability to deliver a sufficient dose of siRNA to the target tissue in vivo hampers the translation of this Nobel prize-winning technology to the clinic. Aptamer-based targeted siRNA delivery merges as a promising approach for cancer-targeted RNAi therapy. By forming a chimera, i.e. the direct link of siRNA with an aptamers, the aptamer acts as a targeting moiety to guide the siRNA to the target cells. Upon binding to the cell surface marker protein, the chimera enters the cells via receptor-mediated endocytosis. Through a yet-to-be elucidated route/mechanism, the siRNA is delivered to the cytoplasm and recognized and processed by Dicer to release the mature siRNA followed by the degradation of target mRNA [16] (Figure 2).

McNamara et al. were among the first to have developed A10-aptamer-siRNA chimera against prostate-specific membrane antigen (PSMA) fused with siRNA to pro-survival genes polo-kinase 1 (PLK1) or B-cell lymphoma-2 (BCL2) [50]. This chimera maintained the aptamer's binding ability and silenced mRNA expressions of Plk1 and Bcl2 with an efficiency of approximately $80 \%$ and $90 \%$ in vitro, respectively. The chimera is internalized into cells via endocytosis where the siRNA is subject to the endogenous Dicer enzyme processing following its incorporation into the RISC, which subsequently targets the antiapoptotic genes and promotion of cell death [50]. After administration of the chimera to mice bearing PSMA-positive xenografts of prostate cancer (200 pmol/mouse every other day for 3 weeks), tumour volumes in control-treated mice increased 3.63-fold, compared to a 2.21-fold reduction in the chimera-treated mice. Also, no obvious morbidity or mortality was observed following the treatment, suggesting that this aptamer-siRNA chimera is not toxic to the animals in these experiments. Subsequently, second generation of A10-aptamer-siRNA chimeras were devised to facilitate chemical synthesis and for better systemic administrationby reducing the size of the aptamer to 32-40 nucleotides and the addition of a $20 \mathrm{kDa}$ polyethylene glycol (PEG) to increase blood residence time [67, 68]. These optimized second-generation chimeras exhibited the same knockdown efficacy at a 100-fold lower 
dosage (4 nM) compared to the first generation chimera. About 70\% PSMA-positive prostate tumours treated with the chimera (250 pmol/mouse, every other day for a total five injections) was regressed and there was a statistically significant reduction in size compared to controls at the end of the treatment. These studies suggest that the aptamer-siRNA chimera system could be a promising approach for targeted delivery of siRNAs for cancer therapeutics.

In addition to the direct fusion of siRNA molecules to aptamers for the specific delivery of RNAi-based therapeutics, siRNAs also can be non-covalently conjugated to aptamers via adapter linkages [69]. Chu and colleagues have non-covalently coupled biotinylated A9 antiPSMA aptamer with functional siRNAs against lamin A/C mRNA or GAPDH through a modular streptavidin linkage [70]. Delivery of aptamer-streptavidin-siRNA conjugates has been found to be an efficient and successful means of knockdowning target gene expression following cellular internalisation into LNCaP cells expressing the PSMA antigens, but not the control PC-3 cells, indicating selective and specific delivery of siRNA to target cells mediated by non-covalently aptamer-siRNA conjugates. Bridging a specific aptamer with a functional siRNA through the non-covalent interaction such as biotin-streptavidin for targeted therapeutics is widely used primarily due to its ease of use and modularity [71, 72]. However, the elicitation of immunogenicity by streptavidin as a non-covalent conjugates limits their usage as for siRNAs delivery in the clinic. As a result, other forms of less immunogenic noncovalent linkages, such as hydrogen bonding, are also being explored [70, 73, 74]. Nonetheless, there are some merits of utilising a non-covalent linkage for delivery of noncoding RNAs (siRNA, shRNA). For instance, prior to entrance to the RISC complex, siRNA should be separated from the conjugated moiety including streptavidin that could be beneficial to further processing of siRNAs [70]. Table 2 summarizes cell-specific aptamers for targeted delivery of siRNA.

\section{Aptamer-miRNA Delivery System}

MiRNA, a small RNA with 20-22 nucleotides, regulates cellular functions such as differentiation, proliferation, apoptosis as well as tumour suppression [81, 82]. It is speculated that modulation of multiple genes simultaneously via manipulating miRNA functions constitutes a promising next generation of anti-cancer therapeutics [83, 84]. However, the possibility of interacting with multiple genes at multiple tissues by a single miRNA demands specific delivery of miRNAs to their targets, in order to minimize unexpected side effects [83]. Therefore, the development of safe and effective strategies for specific delivery of miRNAs is currently under intense investigation. As illustrated in Figure 
2, the aim is to conjugate an aptamer with a miRNA so that the miRNA can be specifically targeted to the cells expressing the ligand recognized by the aptamer. The miRNA gains entry into the cancer cells via aptamer-dependent endocytosis, followed by processing by Dicer and loading of the miRNA duplex into an Argonaut protein in the miRNA-induced silencing complex.

Esposito and colleagues developed a multifunctional aptamer-miRNA conjugate by bridging the tumour suppressor let-7g miRNA to the GL21.T aptamer that specifically bind to the oncogenic receptor tyrosine kinase Axl [85]. They demonstrated that this conjugate could selectively deliver functional let-7g miRNA to Axl-expressing A549 cancer cells and silence let-7g target genes mediated by the RNAi machinery both in vitro and in vivo (1.6 $\mathrm{nmol} /$ mouse, three injections a week for 3 weeks), resulting in inhibition of tumour cell proliferation, migration and survival in vitro and reduction of tumour growth in a xenograft model of lung adenocarcinoma. In addition, Dai et al. explored a MUC1 aptamer-miR-29b chimera (Chi-29b) for increasing chemosensitivity in an OVCAR-3 ovarian cancer model [86]. This is based on the known functions of miR-29b in inducing global DNA hypomethylation and tumour suppressor gene re-expression by directly targeting DNA methyltransferase (Dnmt) 3a and 3b expression and indirectly targeting Dnmt1 expression [87]. As PTEN methylation underlies the inactivation of the PTEN gene in ovarian endometrioid carcinoma and clear cell carcinoma of the ovary [88], gene therapy by overexpression miR-29b may confer enhanced anti-tumour effect. Indeed, intraperitoneal injection (15 $\mu \mathrm{g} /$ mouse, once every two days for 4 weeks) of the chimera to the tumourbearing mice significantly promoted apoptosis in the stepwise developed paclitaxel-resistant OVCAR-3-Taxol cells and achieved a 238-fold reduction in $\mathrm{IC}_{50}$ value compared to normal OVCAR-3 cells with increased apoptosis in the tumour cells. Mechanistically, antichemoresistant function the MUC1 aptamer-miR-29b chimera was attributed to the elevated PTEN expression, downregulation of Akt phosphorylation and upregulation of Bax proapoptotic protein levels.

\section{Aptamer-mediated shRNA Delivery for Cancer Therapy}

ShRNA is an alternative approach to RNAi. Instead of being chemically synthesized as in the case of siRNA, shRNA can be introduced by a number of forms of DNA construct: DNA plasmid, linear template, or packaged retroviral/lentiviral vectors [89, 90]. shRNAs possess several intrinsic advantages compared to siRNA, as generated in host cells, shRNA elicits a more durable gene silencing than that of siRNAs, which are normally degraded around $48 \mathrm{~h}$ 
after the in vivo administration [91]. However, the requirement of the use of gene transfection or viral vector limits the application of shRNA in the clinical settings.

In order to develop vector-free RNAi using shRNA, Ni et al fused 56 nucleotides RNA A103 aptamer targeting PSMA to a 50 nucleotide DNA-activated protein kinase, catalytic polypeptide (DNAPK) shRNA, as DNAPK is a radiosensitisation target [47]. After incubating LNCaP cells with $400 \mathrm{nM}$ chimera for 48 hours, the aptamer-shRNA caused a significant reduction in DNAPK, BRCA2 and ATM mRNA levels. It was confirmed that the A10-3 aptamer-shRNA was internalized into cells and processed by RNAi machinery to yield mature siRNA. Treating subcutaneously implanted LNCaP tumour with radiation plus two intratumoural injections of 200 pmol of A10-3 aptamer-DNAPK shRNA resulted in a dramatically extended time to reach quadruple tumour volume by $~ 10$ weeks compared with one week in tumour treated with control. Thus, aptamer-targeted delivery of shRNA resulted in targeted knockdown of DNAPK, enhanced radiosensitivity and suppressed tumour growth.

\section{Aptamer-functionalised Nanoparticles for Targeted siRNA/miRNA/shRNA Delivery}

Following specific binding to tumour cell receptors and the internalization of aptamer-siRNA chimera, siRNA-mediated silencing of target oncogenes could elicit growth arrest and tumour cell death [70]. However, how such chimeras escape from endosomes/lysosomes after cell entrance remains unclear, which might account for the phenomena that high concentrations of siRNA related chimeras need to be administrated for cancer treatment $[50,75,92]$. To overcome this limitation, recent advances in bionanotechnology enables the conjugation of aptamer-siRNA to nanocarriers such as liposome, gold or other magnetic nanoparticles with endosome-rupturing capabilities [93, 94]. The nanoparticle based siRNA delivery strategy remarkably increased the siRNA payload and held the potential for multifunction improvement.

Bagalkot and Gao have developed a novel two-step approach for linking siRNA-aptamer chimeras to nanoparticles-bearing endosome rupturing capability for conferring siRNA functions [95]. This non-covalently engineering not only assists siRNA removing from the surface of nanoparticles after internalisation but also reduces some of the positive charges of nanoparticle. The later subsequently weekended the interaction between nanoparticles and aptamers, which conferred flexibility to aptamer, improved binding affinity and made it more accessible to target molecules. The approach resulted in a remarkable $34 \%$ improvement in efficiency of gene silencing, compared to the conventional approaches that was based on 
simple mixing of siRNA-aptamer with nontargeted complexes. Another example of noncovalently fusion between aptamer-siRNA with nanoparticles was reported by Zhao et al in that they developed an efficient ALCL cell/tissue-specific carrier system by formulating a functional RNA nanocomplex comprised of the CD30-targeting aptamer and an anaplastic lymphoma kinase (ALK)-targeted siRNA within nano-sized polyethyleneimine (PEI) polymer carriers [96]. Through western blotting analysis and immunocytochemical staining, Karpas 299 cells treated with nanocomplexes displayed an efficient knockdown of the nucelophosmin- ALK (NPM-ALK) fusion protein, but did not affect the $\beta$-actin expression. Consistently, the percentage of apoptotic cells was $14.1 \%$ after $24 \mathrm{~h}$ treatment with nanocomplex, showing a $\sim$-fold increase in cell death compared to the control $(P<0.05)$. Combining siRNAs into nanoparticles increases their size that could prevent the rapid clearance of siRNA from the blood circulation. This system is able to join various types of siRNAs and/or small therapeutic agents to create synergistic effects on cancer therapy. In addition, the positively charged nanocarrier is beneficial for siRNA immobilization and escape from organisms (endosome and lysosome).

In addition to the non-covalent conjugation, RNA aptamers also can be chemically modified to achieve the covalent conjugation to nanovehicles. Kim et al. covalently assembled a 50$\mathrm{NH}_{2}$-modified PSMA aptamer with a polymer containing branched PEI and PEG (PEI-PEG) for co-delivery of Bcl-xL shRNA and doxorubicin [97]. In this study, doxorubicin was directly intercalated into the double-stranded stem of aptamers and the anti-apoptotic factor Bcl-xL shRNA was linked with PEI polymer to form aptamer-conjugated nanoparticles. This combinatorial design synergistically caused LNCaP cell death selectively, compared to the treatment in which two individual therapeutics were simply mixed. In addition to targeted delivery of siRNA/shRNA, miRNAs also can be covalently conjugated to an aptamer functionalised nanoparticle for miRNA-mediated silencing to their target messenger RNAs, resulting in the inhibition of mRNA translation and tumouricidal efficacy [98].

\section{Clinical Trials of Aptamer-mediated Cancer Therapy}

With the encouraging results from preclinical development of aptamer-mediated gene therapy, two aptamer-based cancer therapeutics have progressed to clinical trial stages (Table $\mathbf{3}$ ).

\section{AS1411 Aptamer}


AS1411 is a 26-nucleotide phosphodiester DNA aptamer that targets to the external domain of nucleolin on the membrane, a nuclear matrix protein over-expressed on cell surface of various cancers such as lung cancer, breast cancer, pancreatic cancer, colon cancer, ovarian cancer and leukemia and lymphoma [99]. The nucleolin protein mediates cellular adhesion, endocytosis, and signal transduction and plays important roles in progression of malignant transformation and invasion [99, 100]. The AS1411 aptamer gains the intracellular access when nucleolin is transported from the plasma membrane to the inside of the cell [101]. Blocking of nucleolin by AS1411 contributes to the inhibition of tumour progression both in preclinical and clinical anticancer trials. AS1411 was able to block the activation of canonical nuclear factor- $\kappa \mathrm{B}$ signalling which closely correlates to the chemoresistance of cancers and decrease the binding of nucleolin to Bcl-2 mRNA, resulting in the reduction of Bcl-2 and enhanced anticancer effects [67, 102-104]. AS1411 was the first aptamer approved in clinical trials for treatment of various types of cancer. The phase I clinical trial of AS1411 in patients with various solid tumours revealed promising antitumour activity and a lack of significant systemic toxicity $[100,105,106]$. After a continuous intravenous infusion with a dose escalation from $1 \mathrm{mg} / \mathrm{kg} / \mathrm{d}$ up to $10 \mathrm{mg} / \mathrm{kg} / \mathrm{d}$ in patients with advanced solid tumours, no serious toxicity of AS1411 was observed in 15 patients and 2 patients who received a 4-day and a 7-day infusion, respectively. In addition, no significant clinical toxicity was reported after administration of AS1411 to rats and dogs with IV bolus dosages of up to $100 \mathrm{mg} / \mathrm{kg}$ and $10 \mathrm{mg} / \mathrm{kg} / \mathrm{d}$, respectively [105]. Multi-institutional phase II clinical trials of AS1411 in refractory or relapsed acute myeloid leukaemia (at a dose $40 \mathrm{mg} / \mathrm{kg} / \mathrm{day}$ or $80 \mathrm{mg} / \mathrm{kg} /$ day) and non-small cell lung cancers (NSCLC) are now under way [100, 106, 107]. Most recently, Rosenberg and colleagues examined the efficacy and safety of AS1411 in the anti-cancer treatment of patients with advanced renal cell carcinoma (RCC) who had failed tyrosine kinase inhibitor therapy in a phase II trial [107]. The extensive clinical trial data available so far suggest that AS1411 is a promising candidate for targeted anticancer gene therapy.

\section{NOX-A12 Aptamer}

NOX-A12 is a 45 nucleotide-long L-RNA aptamer with a $40 \mathrm{kDa}$ PEG linkage at the 3terminal developed by Spiegelmer technology on the basis of classic SELEX against the chiral mirror image of the target [108]. NOX-A12 was produced to target stroma cell-derived factor-1 ligand 12 (CXCL12), a chemokine involved in promotion of stem cell migration, tumour metastasis, vascular angiogenesis and tissue regeneration [100, 109]. NOX-A12 aptamers have been shown to possess various intrinsic advantages when used in clinical trials: 
1) they are small molecules and thus facilitate biopharmaceutical manufacture; 2) as Lnucleotides, they are extremely resistant to nuclease mediated degradation and hybridization in cells even without the need for further chemical modification; 3) Spiegelmers are nonimmunogenic and do not activate the cellular immune response; 4) NOX-A12 has therapeutic potential for hematopoietic stem cell transplantation, multiple myelomas and non- Hodgkin's lymphoma. Recently, A Vater and colleagues [110] evaluated white blood cells (WBCs) mobilization in animals (mice and monkeys) and first-in-human clinical study to ascertain safety, pharmacokinetics, and pharmacodynamics of NOX-A12 in healthy volunteers. Both in mice and cynomolgus monkeys, NOX-A12 was superior to the CXCR4 antagonist plerixafor in mobilization of WBCs, hematopoietic stem cells (HSCs) and progenitor cells into peripheral blood (PB) by targeting the chemokine CXCL12. Single dose administration of NOX-A12 (0.05 mg/kg to $10.8 \mathrm{mg} / \mathrm{kg})$ had a benign safety profile in healthy volunteers and also dose-dependently mobilized WBCs and HSCs into PB. At the highest dose $(10.8 \mathrm{mg} / \mathrm{kg})$, NOX-12 had a plasma half-life of $38 \mathrm{~h}$ and a prolonged duration of WBC and HSC mobilization for more than 4 days. HSC peak mobilization reached a plateau at five times the original level at a $5.4 \mathrm{mg} / \mathrm{kg}$ intravenous injection. In this study, NOX-A12 has exhibited a safe and well tolerated profile and demonstrated efficacy in vivo through antagonism of CXCL12 signalling as evident from $\mathrm{WBC}$ and $\mathrm{CD} 34^{+}$cell mobilization under different dose regimen over several days. Studies by independent groups revealed that disruption of CXCL12/CXCR4/CXCR7 signalling pathways could improve cancer therapies [110-113]. Through modulating CXCL12-CXCR4 interactions, NOX-A12 aptamer mobilizes malignant cells away from healthy tissues and functions as a novel anticancer therapeutics for not only hematological cancers but also for a number of malignant cancers. Therefore, NOX-A12 aptamer may provide fresh opportunities to advance conventional chemotherapy or facilitating gene therapy.

\section{CONCLUSIONS AND FUTURE PERSPECTIVES}

Cancer is still one of the leading causes of death world-wide. The outcome of current treatment options for cancer, including surgery, chemo- and/or radio-therapy, is still far from ideal in many cases. Aptamers as a chemical antibody possess great attributes for clinical application both in cancer diagnostics and targeted cancer therapy. In particular, aptamers are emerging as a promising targeting agent for vector-free delivery of therapeutic genes (i.e. siRNA, miRNA and shRNA) for the next generation of cancer gene therapy. Future developments will focus on the selection and/or engineering shorter aptamers for a better 
cost-benefit ratio. Tumour penetration is a vastly under- appreciated and under-studied aspect area in cancer gene therapy, but the extent of tumour penetration throughout the entire tumour is one of the key aspects underlying failure of current cancer drug therapy in solid tumours [114]. The selection of effective aptamers with the smallest possible size through careful post-selection screening and engineering plus the fine tuning of blood residence time and tumour penetration will assist the future development of aptamer-guided gene therapy modalities with superior tumour penetration and thus better clinical outcome. The combination of traditional anticancer therapies along with single or multiple gene therapy regimens may offer more promising outcomes than either of these anticancer approaches alone. With further reduction of potential immune responses and side effects, the vector-free form of aptamer-guided cancer gene therapy will become one of the effective weapons in the anticancer arsenal in the near future.

\section{CONFLICT OF INTEREST}

The author(s) confirm that this article content has no conflicts of interest.

\section{ACKNOWLEDGEMENT}

Work at the authors' laboratory was supported by grants from National Health and Medical Research Council of Australia, Australia-India Strategic Research Fund, Discovery Project grant (DP140100002) from Australian Research Council and The CASS Foundation.

\section{PATIENT CONSENT}

Declared none. 


\section{REFERENCE}

[1] O'Connor ML, Xiang D, Shigdar S, et al. Cancer stem cells: A contentious hypothesis now moving forward. Cancer Lett 2014; 344 (2):180-7.

[2] Cancer Facts \& Figures 2014 [online] 2014 [cited 2014]. Available at: http://www.cancer.org/research/cancerfactsstatistics/cancerfactsfigures2014/.

[3] Bhatia S, Menezes ME, Das SK, et al. Innovative approaches for enhancing cancer gene therapy. Discov Med 2013; 15 (84):309-17.

[4] Kaufmann KB, Buning H, Galy A, Schambach A, Grez M. Gene therapy on the move. EMBO Mol Med 2013; 5 (11):1642-61.

[5] Kircheis R, Wightman L, Kursa M, Ostermann E, Wagner E. Tumor-targeted gene delivery: an attractive strategy to use highly active effector molecules in cancer treatment. Gene Ther 2002; 9 (11):731-5.

[6] Huang TG, Savontaus MJ, Shinozaki K, Sauter BV, Woo SLC. Telomerase-dependent oncolytic adenovirus for cancer treatment. Gene Ther 2003; 10 (15):1241-7.

[7] Thomas CE, Ehrhardt A, Kay MA. Progress and problems with the use of viral vectors for gene therapy. Nat Rev Genet 2003; 4 (5):346-58.

[8] Ding B, Li T, Zhang J, Zhao L, Zhai G. Advances in liver-directed gene therapy for hepatocellular carcinoma by non-viral delivery systems. Curr Gene Ther 2012; 12 (2):92-102.

[9] Kay MA. State-of-the-art gene-based therapies: the road ahead. Nat Rev Genet 2011; 12 (5):316-28.

[10] Doggrell SA. Pegaptanib: the first antiangiogenic agent approved for neovascular macular degeneration. Expert Opin Pharmacother 2005; 6 (8):1421-3.

[11] Hicke BJ, Stephens AW. Escort aptamers: a delivery service for diagnosis and therapy. J Clin Invest 2000; 106 (8):923-8.

[12] Shigdar S, Lin J, Li Y, et al. Cancer stem cell targeting: the next generation of cancer therapy and molecular imaging. Ther Deliv 2012; 3 (2):227-44.

[13] Shigdar S, Ward AC, De A, Yang CJ, Wei M, Duan W. Clinical applications of aptamers and nucleic acid therapeutics in haematological malignancies. Br J Haematol 2011; 155 (1):3-13.

[14] Phillips JA, Lopez-Colon D, Zhu Z, Xu Y, Tan W. Applications of aptamers in cancer cell biology. Anal Chim Acta 2008; 621 (2):101-8.

[15] Que-Gewirth NS, Sullenger BA. Gene therapy progress and prospects: RNA aptamers. Gene Ther 2007; 14 (4):283-91.

[16] Zhou J, Bobbin ML, Burnett JC, Rossi JJ. Current progress of RNA aptamer-based therapeutics. Front Genet 2012; 3234.

[17] Verma IM. Medicine. Gene therapy that works. Science 2013; 341 (6148):853-5.

[18] Heo DS. Progress and limitations in cancer gene therapy. Genet Med 2002; 4 (6 Suppl):52S-5S.

[19] El-Aneed A. Current strategies in cancer gene therapy. Eur J Pharmacol 2004; 498 (1-3):1-8.

[20] Walther W, Schlag PM. Current status of gene therapy for cancer. Curr Opin Oncol 2013; 25 (6):659-64.

[21] Shi J, Zheng D. An update on gene therapy in China. Curr Opin Mol Ther 2009; 11 (5):547-53.

[22] Liang M. Clinical development of oncolytic viruses in China. Curr Pharm Biotechnol 2012; 13 (9):1852-7.

[23] Romano G. Development of safer gene delivery systems to minimize the risk of insertional mutagenesisrelated malignancies: a critical issue for the field of gene therapy. ISRN Oncol 2012; 2012616310.

[24] Murakami T, Sunada Y. Plasmid DNA gene therapy by electroporation: principles and recent advances. Curr Gene Ther 2011; 11 (6):447-56.

[25] Gao Y, Liu XL, Li XR. Research progress on siRNA delivery with nonviral carriers. Int J Nanomedicine 2011; 6 1017-25.

[26] Liu HY, Gao X. A universal protein tag for delivery of SiRNA-aptamer chimeras. Sci Rep 2013; 33129.

[27] Shen X, Storm T, Kay MA. Characterization of the relationship of AAV capsid domain swapping to liver transduction efficiency. Mol Ther 2007; 15 (11):1955-62.

[28] Greten TF, Korangy F, Manns MP, Malek NP. Molecular therapy for the treatment of hepatocellular carcinoma. Br J Cancer 2009; 100 (1):19-23.

[29] Thomas MB, Jaffe D, Choti MM, et al. Hepatocellular carcinoma: consensus recommendations of the National Cancer Institute Clinical Trials Planning Meeting. J Clin Oncol 2010; 28 (25):3994-4005.

[30] Ellington AD, Szostak JW. In vitro selection of RNA molecules that bind specific ligands. Nature 1990; 346 (6287):818-22. 
[31] Tuerk C, Gold L. Systematic evolution of ligands by exponential enrichment: RNA ligands to bacteriophage T4 DNA polymerase. Science 1990; 249 (4968):505-10.

[32] Shangguan D, Tang Z, Mallikaratchy P, Xiao Z, Tan W. Optimization and modifications of aptamers selected from live cancer cell lines. Chembiochem 2007; 8 (6):603-6.

[33] Cerchia L, Hamm J, Libri D, Tavitian B, de Franciscis V. Nucleic acid aptamers in cancer medicine. FEBS Lett 2002; 528 (1-3):12-6.

[34] White RR, Sullenger BA, Rusconi CP. Developing aptamers into therapeutics. J Clin Invest 2000; 106 (8):929-34.

[35] Drolet DW, Nelson J, Tucker CE, et al. Pharmacokinetics and safety of an anti-vascular endothelial growth factor aptamer (NX1838) following injection into the vitreous humor of rhesus monkeys. Pharm Res $2000 ; 17$ (12):1503-10.

[36] Shigdar S, Macdonald J, O'Connor M, et al. Aptamers as theranostic agents: modifications, serum stability and functionalisation. Sensors (Basel) 2013; 13 (10):13624-37.

[37] Keefe AD, Pai S, Ellington A. Aptamers as therapeutics. Nat Rev Drug Discov 2010; 9 (7):537-50.

[38] Healy JM, Lewis SD, Kurz M, et al. Pharmacokinetics and biodistribution of novel aptamer compositions. Pharm Res 2004; 21 (12):2234-46.

[39] Zhao Z, Xu L, Shi X, Tan W, Fang X, Shangguan D. Recognition of subtype non-small cell lung cancer by DNA aptamers selected from living cells. Analyst 2009; 134 (9):1808-14.

[40] Kaur H, Li JJ, Bay B-H, Yung L-YL. Investigating the antiproliferative activity of high affinity DNA aptamer on cancer cells. PLoS One 2013; 8 (1):e50964.

[41] Lee YJ, Han SR, Kim NY, Lee S-H, Jeong J-S, Lee S-W. An RNA aptamer that binds carcinoembryonic antigen inhibits hepatic metastasis of colon cancer cells in mice. Gastroenterology 2012; 143 (1):155-65.e8.

[42] Roth F, De La Fuente AC, Vella JL, Zoso A, Inverardi L, Serafini P. Aptamer-mediated blockade of IL4Ralpha triggers apoptosis of MDSCs and limits tumor progression. Cancer Res 2012; 72 (6):1373-83.

[43] Mern DS, Hasskarl J, Burwinkel B. Inhibition of Id proteins by a peptide aptamer induces cell-cycle arrest and apoptosis in ovarian cancer cells. Br J Cancer 2010; 103 (8):1237-44.

[44] Mern DS, Hoppe-Seyler K, Hoppe-Seyler F, Hasskarl J, Burwinkel B. Targeting Id1 and Id3 by a specific peptide aptamer induces E-box promoter activity, cell cycle arrest, and apoptosis in breast cancer cells. Breast Cancer Res Treat 2010; 124 (3):623-33.

[45] Thiel KW, Giangrande PH. Intracellular delivery of RNA-based therapeutics using aptamers. Ther Deliv 2010; 1 (6):849-61.

[46] Cerchia L, Esposito CL, Camorani S, Catuogno S, Franciscis Vd. Coupling Aptamers to Short Interfering RNAs as Therapeutics. Pharmaceuticals 2011; 4 (12):1434-49.

[47] Ni X, Zhang Y, Ribas J, et al. Prostate-targeted radiosensitization via aptamer-shRNA chimeras in human tumor xenografts. J Clin Invest 2011; 121 (6):2383-90.

[48] Farokhzad OC, Jon S, Khademhosseini A, Tran T-NT, Lavan DA, Langer R. Nanoparticle-aptamer bioconjugates: a new approach for targeting prostate cancer cells. Cancer Res 2004; 64 (21):7668-72.

[49] Chu TC, Marks JW, 3rd, Lavery LA, et al. Aptamer:toxin conjugates that specifically target prostate tumor cells. Cancer Res 2006; 66 (12):5989-92.

[50] McNamara JO, 2nd, Andrechek ER, Wang Y, et al. Cell type-specific delivery of siRNAs with aptamersiRNA chimeras. Nat Biotechnol 2006; 24 (8):1005-15.

[51] Ruff P, Koh KD, Keskin H, Pai RB, Storici F. Aptamer-guided gene targeting in yeast and human cells. Nucleic Acids Res 2014; 42 (7):e61.

[52] FDA Approves Nexavar for Patients with Inoperable Liver Cancer [online] November 19, 2007 [cited November 19, 2007]. Available at: http://www.fda.gov/NewsEvents/Newsroom/PressAnnouncements/2007/ucm109030.htm.

[53] Bhattacharya SD, Mi Z, Kim VM, Guo H, Talbot LJ, Kuo PC. Osteopontin regulates epithelial mesenchymal transition-associated growth of hepatocellular cancer in a mouse xenograft model. Ann Surg 2012; 255 (2):319-25.

[54] SGI-110 in the Treatment of Advanced Hepatocellular Carcinoma (HCC) [online] January 17, 2014 [cited January 17, 2014]. Available at: http://clinicaltrials.gov/ct2/show/record/NCT01752933.

[55] Sun B-S, Dong Q-Z, Ye Q-H, et al. Lentiviral-mediated miRNA against osteopontin suppresses tumor growth and metastasis of human hepatocellular carcinoma. Hepatology 2008; 48 (6):1834-42.

[56] Mi Z, Bhattacharya SD, Kim VM, Guo H, Talbot LJ, Kuo PC. Osteopontin promotes CCL5-mesenchymal stromal cell-mediated breast cancer metastasis. Carcinogenesis 2011; 32 (4):477-87. 
[57] Cheng J, Wang W, Sun C, Li M, Wang B, Lv Y. Meta-Analysis of the Prognostic and Diagnostic Significance of Serum/Plasma Osteopontin in Hepatocellular Carcinoma. J Clin Gastroenterol 2013;

[58] Dong Q-Z, Zhang X-F, Zhao Y, et al. Osteopontin promoter polymorphisms at locus -443 significantly affect the metastasis and prognosis of human hepatocellular carcinoma. Hepatology 2013; 57 (3):1024-34.

[59] Zhou C, Zhou HJ, Zhang XF, et al. Postoperative serum osteopontin level is a novel monitor for treatment response and tumor recurrence after resection of hepatitis B-related hepatocellular carcinoma. Ann Surg Oncol 2013; 20 (3):929-37.

[60] Zhu W, Guo L, Zhang B, et al. Combination of Osteopontin with Peritumoral Infiltrating Macrophages is Associated with Poor Prognosis of Early-Stage Hepatocellular Carcinoma after Curative Resection. Ann Surg Oncol 2013;

[61] Zhao J, Dong L, Lu B, et al. Down-regulation of osteopontin suppresses growth and metastasis of hepatocellular carcinoma via induction of apoptosis. Gastroenterology 2008; 135 (3):956-68.

[62] Mi Z, Guo H, Russell MB, Liu Y, Sullenger BA, Kuo PC. RNA aptamer blockade of osteopontin inhibits growth and metastasis of MDA-MB231 breast cancer cells. Mol Ther 2009; 17 (1):153-61.

[63] Hammarstrom S. The carcinoembryonic antigen (CEA) family: structures, suggested functions and expression in normal and malignant tissues. Semin Cancer Biol 1999; 9 (2):67-81.

[64] Jones C, Badger SA, Epanomeratikis E, McKie LD, Diamond T, Taylor MA. Role of carcinoembryonic antigen as a marker for colorectal liver metastases. Br J Biomed Sci 2013; 70 (2):47-50.

[65] Zimmer R, Thomas P. Mutations in the carcinoembryonic antigen gene in colorectal cancer patients: implications on liver metastasis. Cancer Res 2001; 61 (7):2822-6.

[66] Bumcrot D, Manoharan M, Koteliansky V, Sah DWY. RNAi therapeutics: a potential new class of pharmaceutical drugs. Nat Chem Biol 2006; 2 (12):711-9.

[67] Soundararajan S, Chen W, Spicer EK, Courtenay-Luck N, Fernandes DJ. The nucleolin targeting aptamer AS1411 destabilizes Bcl-2 messenger RNA in human breast cancer cells. Cancer Res 2008; 68 (7):2358-65.

[68] Dassie JP, Liu XY, Thomas GS, et al. Systemic administration of optimized aptamer-siRNA chimeras promotes regression of PSMA-expressing tumors. Nat Biotechnol 2009; 27 (9):839-49.

[69] Zhou J, Rossi JJ. Aptamer-targeted cell-specific RNA interference. Silence 2010; 1 (1):4.

[70] Chu TC, Twu KY, Ellington AD, Levy M. Aptamer mediated siRNA delivery. Nucleic Acids Res 2006; 34 (10): 73.

[71] Zhang Y, Pardridge WM. Conjugation of brain-derived neurotrophic factor to a blood-brain barrier drug targeting system enables neuroprotection in regional brain ischemia following intravenous injection of the neurotrophin. Brain Res 2001; 889 (1-2):49-56.

[72] Schnyder A, Krahenbuhl S, Drewe J, Huwyler J. Targeting of daunomycin using biotinylated immunoliposomes: pharmacokinetics, tissue distribution and in vitro pharmacological effects. J Drug Target 2005; 13 (5):325-35.

[73] Breitz HB, Weiden PL, Beaumier PL, et al. Clinical optimization of pretargeted radioimmunotherapy with antibody-streptavidin conjugate and 90Y-DOTA-biotin. J Nucl Med 2000; 41 (1):131-40.

[74] Meyer DL, Schultz J, Lin Y, et al. Reduced antibody response to streptavidin through site-directed mutagenesis. Protein Sci 2001; 10 (3):491-503.

[75] Dassie JP, Liu X-Y, Thomas GS, et al. Systemic administration of optimized aptamer-siRNA chimeras promotes regression of PSMA-expressing tumors. Nat Biotechnol 2009; 27 (9):839-49.

[76] Zhang X, Liang H, Tan Y, Wu X, Li S, Shi Y. A U87-EGFRvIII cell-specific aptamer mediates small interfering RNA delivery. Biomed Rep 2014; 2 (4):495-9.

[77] Berezhnoy A, Castro I, Levay A, Malek TR, Gilboa E. Aptamer-targeted inhibition of mTOR in T cells enhances antitumor immunity. J Clin Invest 2014; 124 (1):188-97.

[78] Lai W-Y, Wang W-Y, Chang Y-C, Chang C-J, Yang P-C, Peck K. Synergistic inhibition of lung cancer cell invasion, tumor growth and angiogenesis using aptamer-siRNA chimeras. Biomaterials 2014; 35 (9):290514.

[79] Li L, Hou J, Liu X, et al. Nucleolin-targeting liposomes guided by aptamer AS1411 for the delivery of siRNA for the treatment of malignant melanomas. Biomaterials 2014; 35 (12):3840-50.

[80] Yoo H, Jung H, Kim SA, Mok H. Multivalent comb-type aptamer-siRNA conjugates for efficient and selective intracellular delivery. Chem Commun (Camb) 2014; 50 (51):6765-7.

[81] Iorio MV, Croce CM. MicroRNAs in cancer: small molecules with a huge impact. J Clin Oncol 2009; 27 (34):5848-56.

[82] Zamore PD, Haley B. Ribo-gnome: the big world of small RNAs. Science 2005; 309 (5740):1519-24. 
[83] Iorio MV, Croce CM. MicroRNA dysregulation in cancer: diagnostics, monitoring and therapeutics. A comprehensive review. EMBO Mol Med 2012; 4 (3):143-59.

[84] Garofalo M, Condorelli GL, Croce CM, Condorelli G. MicroRNAs as regulators of death receptors signaling. Cell Death Differ 2010; 17 (2):200-8.

[85] Esposito CL, Cerchia L, Catuogno S, et al. Multifunctional Aptamer-miRNA Conjugates for Targeted Cancer Therapy. Mol Ther 2014;

[86] Dai F, Zhang Y, Zhu X, Shan N, Chen Y. The anti-chemoresistant effect and mechanism of MUC1 aptamer-miR-29b chimera in ovarian cancer. Gynecol Oncol 2013; 131 (2):451-9.

[87] Garzon R, Liu S, Fabbri M, et al. MicroRNA-29b induces global DNA hypomethylation and tumor suppressor gene reexpression in acute myeloid leukemia by targeting directly DNMT3A and 3B and indirectly DNMT1. Blood 2009; 113 (25):6411-8.

[88] Sato N, Tsunoda H, Nishida M, et al. Loss of heterozygosity on 10q23.3 and mutation of the tumor suppressor gene PTEN in benign endometrial cyst of the ovary: possible sequence progression from benign endometrial cyst to endometrioid carcinoma and clear cell carcinoma of the ovary. Cancer Res 2000; 60 (24):7052-6.

[89] Yu J-Y, DeRuiter SL, Turner DL. RNA interference by expression of short-interfering RNAs and hairpin RNAs in mammalian cells. Proc Natl Acad Sci U S A 2002; 99 (9):6047-52.

[90] Wang Z, Rao DD, Senzer N, Nemunaitis J. RNA interference and cancer therapy. Pharm Res 2011; 28 (12):2983-95.

[91] Rao DD, Vorhies JS, Senzer N, Nemunaitis J. siRNA vs. shRNA: similarities and differences. Adv Drug Deliv Rev 2009; 61 (9):746-59.

[92] Levy-Nissenbaum E, Radovic-Moreno AF, Wang AZ, Langer R, Farokhzad OC. Nanotechnology and aptamers: applications in drug delivery. Trends Biotechnol 2008; 26 (8):442-9.

[93] Meng H, Liong M, Xia T, et al. Engineered design of mesoporous silica nanoparticles to deliver doxorubicin and P-glycoprotein siRNA to overcome drug resistance in a cancer cell line. ACS Nano 2010; 4 (8):4539-50.

[94] Xia T, Kovochich M, Liong M, et al. Polyethyleneimine coating enhances the cellular uptake of mesoporous silica nanoparticles and allows safe delivery of siRNA and DNA constructs. ACS Nano 2009; 3 (10):3273-86.

[95] Bagalkot V, Gao X. siRNA-aptamer chimeras on nanoparticles: preserving targeting functionality for effective gene silencing. ACS Nano 2011; 5 (10):8131-9.

[96] Zhao N, Bagaria HG, Wong MS, Zu Y. A nanocomplex that is both tumor cell-selective and cancer genespecific for anaplastic large cell lymphoma. J Nanobiotechnology 2011; 92.

[97] Kim E, Jung Y, Choi H, et al. Prostate cancer cell death produced by the co-delivery of Bcl-xL shRNA and doxorubicin using an aptamer-conjugated polyplex. Biomaterials 2010; 31 (16):4592-9.

[98] Wu X, Ding B, Gao J, et al. Second-generation aptamer-conjugated PSMA-targeted delivery system for prostate cancer therapy. Int J Nanomedicine 2011; 6 1747-56.

[99] Bates PJ, Laber DA, Miller DM, Thomas SD, Trent JO. Discovery and development of the G-rich oligonucleotide AS1411 as a novel treatment for cancer. Exp Mol Pathol 2009; 86 (3):151-64.

[100] Sundaram P, Kurniawan H, Byrne ME, Wower J. Therapeutic RNA aptamers in clinical trials. Eur J Pharm Sci 2013; 48 (1-2):259-71.

[101] Soundararajan S, Wang L, Sridharan V, et al. Plasma membrane nucleolin is a receptor for the anticancer aptamer AS1411 in MV4-11 leukemia cells. Mol Pharmacol 2009; 76 (5):984-91.

[102] Girvan AC, Teng Y, Casson LK, et al. AGRO100 inhibits activation of nuclear factor-kappaB (NFkappaB) by forming a complex with NF-kappaB essential modulator (NEMO) and nucleolin. Mol Cancer Ther 2006; 5 (7):1790-9.

[103] Nakanishi C, Toi M. Nuclear factor-kappaB inhibitors as sensitizers to anticancer drugs. Nat Rev Cancer 2005; 5 (4):297-309.

[104] Di Segni A, Farin K, Pinkas-Kramarski R. Identification of nucleolin as new ErbB receptors- interacting protein. PLoS One 2008; 3 (6):e2310.

[105] Ireson CR, Kelland LR. Discovery and development of anticancer aptamers. Mol Cancer Ther 2006; 5 (12):2957-62.

[106] Esposito CL, Catuogno S, de Franciscis V, Cerchia L. New insight into clinical development of nucleic acid aptamers. Discov Med 2011; 11 (61):487-96.

[107] Rosenberg JE, Bambury RM, Van Allen EM, et al. A phase II trial of AS1411 (a novel nucleolin-targeted DNA aptamer) in metastatic renal cell carcinoma. Invest New Drugs 2014; 32 (1):178-87. 
[108] Nolte A, Klussmann S, Bald R, Erdmann VA, Furste JP. Mirror-design of L-oligonucleotide ligands binding to L-arginine. Nat Biotechnol 1996; 14 (9):1116-9.

[109] Sayyed SG, Hagele H, Kulkarni OP, et al. Podocytes produce homeostatic chemokine stromal cellderived factor-1/CXCL12, which contributes to glomerulosclerosis, podocyte loss and albuminuria in a mouse model of type 2 diabetes. Diabetologia 2009; 52 (11):2445-54.

[110] Vater A, Sahlmann J, Kroger N, et al. Hematopoietic stem and progenitor cell mobilization in mice and humans by a first-in-class mirror-image oligonucleotide inhibitor of CXCL12. Clin Pharmacol Ther 2013; 94 (1):150-7.

[111] Domanska UM, Kruizinga RC, Nagengast WB, et al. A review on CXCR4/CXCL12 axis in oncology: no place to hide. Eur J Cancer 2013; 49 (1):219-30.

[112] Kryczek I, Lange A, Mottram P, et al. CXCL12 and vascular endothelial growth factor synergistically induce neoangiogenesis in human ovarian cancers. Cancer Res 2005; 65 (2):465-72.

[113] Burger JA, Peled A. CXCR4 antagonists: targeting the microenvironment in leukemia and other cancers. Leukemia 2009; 23 (1):43-52.

[114] Minchinton AI, Tannock IF. Drug penetration in solid tumours. Nat Rev Cancer 2006; 6 (8):583-92. 
Table 1 Comparison of Key Features between Aptamer and Antibody

\begin{tabular}{|l|l|l|}
\hline & Aptamer & Antibody \\
\hline Size & $\sim 2 \mathrm{~nm}$ & $\sim 15 \mathrm{~nm}$ \\
\hline Generation & In vitro SELEX & In vivo biological system \\
\hline Affinity & High & High \\
\hline Specificity & High & High \\
\hline $\begin{array}{l}\text { Chemical } \\
\text { modification }\end{array}$ & Easy and straightforward & Limited \\
\hline Targets & $\begin{array}{l}\text { Wide range: nucleic acids, peptides, } \\
\text { proteins, organic and inorganic } \\
\text { molecules, a whole cell, entire organ }\end{array}$ & Limited to immunogenic molecule \\
\hline Immunogenicity & No or low & Yes \\
\hline Batch to batch & No or low & Significant \\
\hline Cost & Becoming low with new technology & Low \\
\hline Stability & $\begin{array}{l}\text { Stable across a wide range of storage } \\
\text { and temperature conditions }\end{array}$ & Low \\
\hline & & \\
\hline
\end{tabular}

Table 2 Application of Cell-specific Aptamers for Targeted Delivery of SiRNA

\begin{tabular}{|l|l|l|l|}
\hline Aptamer & Target gene & Cancer & Research Group \\
\hline RNA aptamer & PLK1 and BCL2 & Prostate cancer & McNamara et al. 2006 [50] \\
\hline RNA aptamer & lamin A/C mRNA & Prostate cancer & Chu et al. 2006 [70] \\
\hline RNA aptamer & PLK1 & Prostate cancer & Liu et al. 2013 [26] \\
\hline RNA aptamer & PLK1 or BCL2 & Prostate cancer & Dassie et al. 2009 [75] \\
\hline RNA aptamer & EGFRvIII & Glioblastoma & Zhang et al. 2014 [76] \\
\hline RNA aptamer & mTORC1 & Melanoma, Breast cancer & Berezhnoy et al. 2014 [77] \\
\hline RNA aptamer & SLUG and NRP1 & Lung cancer & Lai et al. 2014 [78] \\
\hline RNA aptamer & BRAF & Melanoma & Li et al. 2014 [79] \\
\hline DNA aptamer & MUC1 & Adenocarcinomas & Yoo et al. 2014 [80] \\
\hline
\end{tabular}

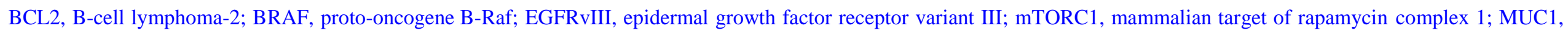
cell surface associated; NRP1, neuropilin 1; PLK1, pro-survival genes polo-kinase 1; SLUG, snail family zinc finger. 
Table 3 Clinical Trials of Aptamer-Based Anti-Cancer Therapies

\begin{tabular}{|c|c|c|c|c|c|c|c|c|c|}
\hline & & & & & \multicolumn{3}{|c|}{ Eligibility } & & \\
\hline NCT number & Drug (Target) & Condition & Status & Phase & Ages (years) & $\begin{array}{l}\text { Genders for } \\
\text { Study }\end{array}$ & $\begin{array}{l}\text { Accepts Healthy } \\
\text { Volunteers }\end{array}$ & Locations & Sponsor \\
\hline NCT01034410 & $\begin{array}{l}\text { AS1411 } \\
\text { (Nucleolin) }\end{array}$ & AML & Completed & 2 & $18-70$ & Both & No & $\begin{array}{l}\text { U.S, Australia, New } \\
\text { Zealand, Taiwan }\end{array}$ & Antisoma Research \\
\hline NCT01194934 & $\begin{array}{l}\text { NOX-A12 } \\
\text { (CXCL12/SD } \\
\text { F-1) }\end{array}$ & HSCT & Completed & 1 & $18-55$ & Both & Yes & Germany & Noxxon Pharma AG \\
\hline
\end{tabular}

AML: Acute Myeloid Leukemia, HSCT: Hematopoietic Stem Cell Transplantation 


\section{Figures Legends:}

Fig. (1). Schematic Representation of RNA Aptamers Generation by SELEX. Specific targets of interest (cells or proteins) are incubated with a pool of single-stranded RNA oligonucleotides with random sequences, followed by the extraction of bound sequences and removal of unbound RNAs. The selected sequences can be subjected to rounds of negative selection by incubating with negative control cells or proteins. The unbounded oligonucleotides are eluted and amplified by RTPCR, followed by next rounds of selection. After the last round of selection (typically round 6-10), the remaining RNA aptamers are converted to DNA and subjected to next generation sequencing or conventional cloning followed by characterization and further engineering.

Fig. (2). Aptamer-Mediated Cancer Gene Therapy Pathways. Therapeutic nucleotides such as siRNA, miRNA and anti-miRNA can be directly conjugated with aptamers or encapsulated within nanoparticles followed by aptamer functionalization, in which aptamer acts as targeted-delivery modalities. Following the specific binding of aptamers to the cell surface targets, both of aptamer conjugated therapeutic nucleotides or nucleotides-loaded nano-carriers internalise into target cancer cells via receptor-mediated endocytosis. After the entrance and escape from endosomes, the released siRNA/miRNA/anti-miRNA in the cytoplasm are cleaved by Dicer into mature siRNA/miRNA/anti-miRNA. These mature nucleotides are unwound and subsequently incorporated into the RNA-induced silencing complex (RISC) system. The guide strand enables the mature siRNA/miRNA/anti-miRNA to recognise target sequences of mRNA, leading to mRNA degradation or translation promotion or repression of specific RNA targets. Part of mature miRNAs are transported into nuclei and interact with their target nucleic acids or genomic DNAs to elicit translation promotion/repression or DNA binding activation/repression. 